\title{
MEDULLOBLASTOMA IN ADULTHOOD
}

\author{
DRAGOȘ HORȘIA ${ }^{1}$ \\ I "Lucian Blaga” University of Sibiu
}

\begin{abstract}
Keywords: $\quad$ tumour, malignancy potential, medulloblastoma, childhood, percentage decreasing, Turcot syndrome, basal cell carcinoma syndrome

Abstract: Defined as a tumour with increased malignancy potential in childhood, medulloblastoma was first reported in the literature by Percival Bailey and Harvey Cushing in 1925. Scientific studies over the years have shown that this type of tumour represents about $20 \%$ of all intracranial tumours encountered in childhood, their percentage decreasing with advancing age. The genetic factor plays an important part in the appearance of medulloblastoma; there are certain diseases, in the patient's history, that can be associated with this type of tumour. Here, we can specify Turcot syndrome (an autosomal recessive disease, rarely encountered) or basal cell carcinoma syndrome. This article presents the case of a young patient (41-year-old) suffering from a cerebellar tumour formation that turned out to be, after histopathological examination, a medulloblastoma. In practice we can find several types of medulloblastoma (desmoplastic or nodular, anaplastic, classical or undifferentiated). In what follows I will try to highlight a few aspects of a classic medulloblastoma.
\end{abstract}

\section{INTRODUCTION}

Medulloblastoma $(1,2,3,4,5,6)$ is the most common intracranial tumour found in children. It occurs less frequently in young people and is exceptional in adults. Of particular interest is the cerebellum $(7,8)$, but cases with hemisphere damage have been described. Male involvement is more common, in a 2:1 ratio compared to females. It grows rapidly and metastasizes in the cervical lymph nodes or at the bone level. The treatment is exclusively surgical and consists in the total excision of this tumour formation. Survival, at five years, is between 40 and $65 \%$. In the presence of metastases, the prognosis falls below 40\%. Optical microscopy and immunohistochemistry are particularly important in the diagnosis of medulloblastoma.

CASE REPORT

I want to present the case of a 41-year-old patient without a significant personal pathological history, presenting with a headache that begins about 6 months before. The craniocerebral CT performed revealed the presence of a left cerebellar tissue formation. Following the neurological examination performed after hospitalization in the Neurosurgery Department, the following were found: cooperating patient, no signs of meningeal irritation, negative paresis tests, examination of cranial nerves without pathological changes, headache syndrome, no disorders of objective sensitivity or mobility, gnosis (can recognize objects with the help of the sense organs), praxis (adaptation of human motor movements for a purpose) within normal limits. Contrast-enhanced brain MRI detects the presence of a left intracerebellar expansive process. In the clinical and imaging context, the neurosurgical intervention is proposed, which aims to ablate the tumour formation. For the ablation of the tumour formation, under general anesthesia, neurosurgical intervention is performed, practicing the incision of the skin in the left occipital region, left occipital craniotomy, ablation of the left cerebellar tumour formation and restoration of the bone flap. After the surgery on the Neurosurgery Department, multiple biopsy fragments were taken and sent to the Pathological Anatomy, the smallest with the dimensions of $5 / 5 / 3 \mathrm{~mm}$, the largest with the dimensions of $25 / 16 / 10 \mathrm{~mm}$. At the end of the surgery, the patient is transferred to the Anesthesia and Intensive Care Neurosurgery department for supervision. Following the establishment of favourable evolutionary behaviour, she is transferred to a neurosurgery salon. The subsequent evolution, under treatment, is a favourable one with the amelioration of the neurological symptomatology. The native cranial MDCT examination (computed tomography with multiple detectors) performed later reveals a left cerebellar postoperative hypodense area with pneumocephalus at this level and adjacent occipital. At discharge, the patient is conscious, cooperative, oriented, in good general condition, feverish, with neurological symptoms in amelioration, with clean surgical wound, without signs of Celsius, which requires care that involves dressing with betadine solution, the wound being closed with sutures to the skin.

\section{DISCUSSIONS}

\section{Macroscopic:}

Medulloblastoma is, macroscopically, a gray-yellow, fleshy, soft consistency, richly vascularized, intraneuroaxial formation, with areas of necrosis. It is poorly delimited.

Microscopic examination revealed a syncytial hypercellular tumour proliferation, consisting of dense groups of small (9) (figure no. 1), undifferentiated cells (10) (figure no 2), round - oval, some elongated with basophilic cytoplasm (figure no. 3), round-oval nucleus or „,carrot shaped”, hyperchromic (11), with granular and fine chromatin located at the periphery. This typical blue staining (hematoxylin-eosin staining) is due to nuclear agglomeration. The presence of Homer-Wright rosettes

${ }^{1}$ Corresponding author: Dragoș Horşia, Str. Ana Ipatescu, Nr. 67, Ap. 5, Sibiu, România, E-mail: dragos.horsia@ulbsibiu.ro, Phone: +40751026442 Article received on 16.06.2021 and accepted for publication on 27.08.2021 


\section{CLINICAL ASPECTS}

$(12,13,14,15)$ (figures no. 4,5$)$ is also highlighted. Homer Wright rosettes are pathognomonic elements for this tumour and consist of the presence of tumour cells arranged around a lumen. The cells have variable shapes and sizes (round, oval or elongated), having a uniform arrangement. There are also situations in which Homer-Wright rosettes are less represented. These formations may be associated with increased mitotic $(16,17,18,19,20)$ activity and high nuclear pleomorphism.

Figure no. 1. Dense groups of small, blue, round-oval cells with sheets arrangement (present throughout the microscopic field) (H.E. x.10)

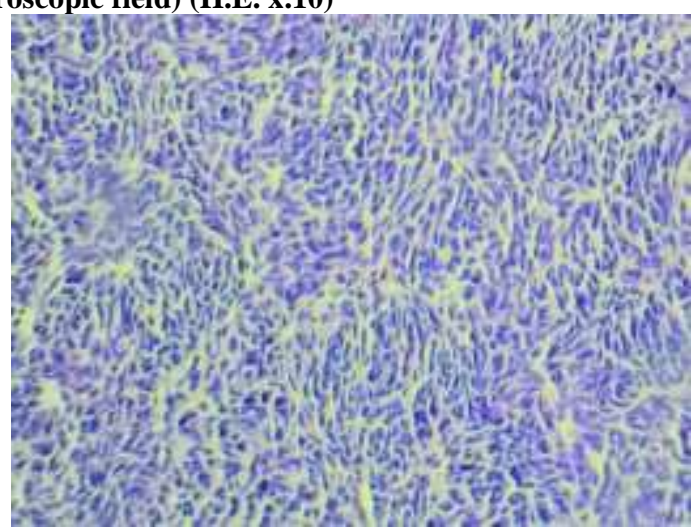

Figure no. 2. Dense groups of undifferentiated, blue cells (due to a nuclear agglomeration) with the presence of numerous mitoses (H.E. x.20)

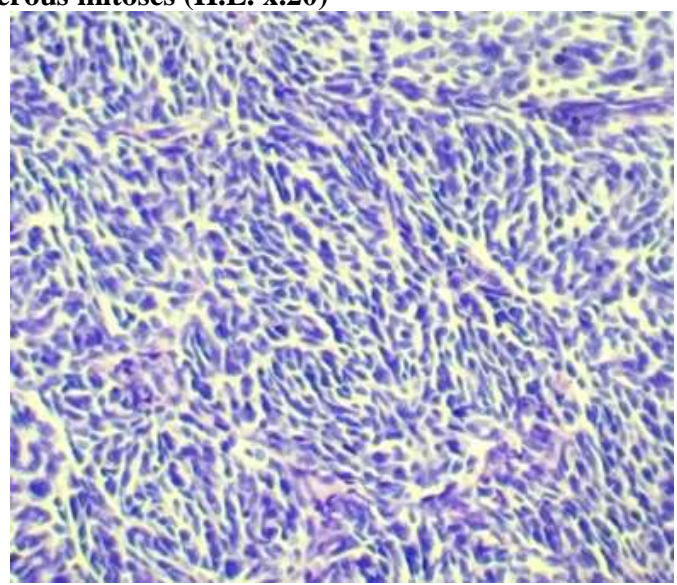

Figure no. 3. Some elongated cells - Having a basophilic cytoplasm, with round-oval nucleus commonly found in this type of malignant tumor. (indicated by dark arrows)(H.E. $x$ 40)

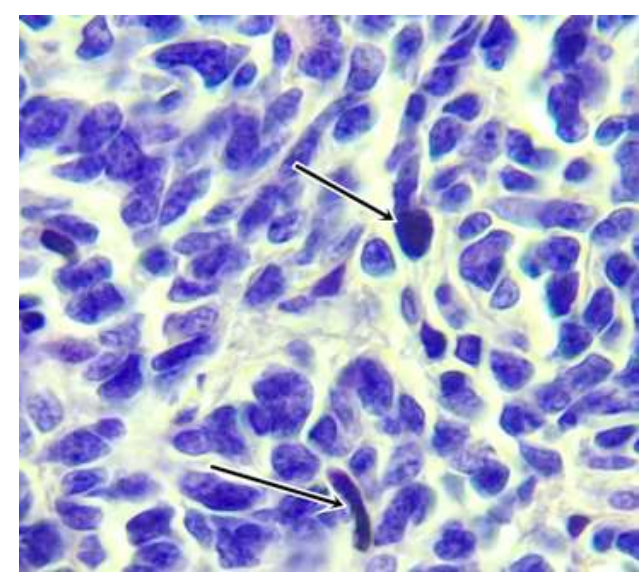

Figure no. 4. Homer-Wright rosettes - Pathognomonic formations of variable size formed by cells of relatively large size, arranged uniformly, annularly, surrounding acellular areas (indicated by dark arrows) (H.E. x 20)

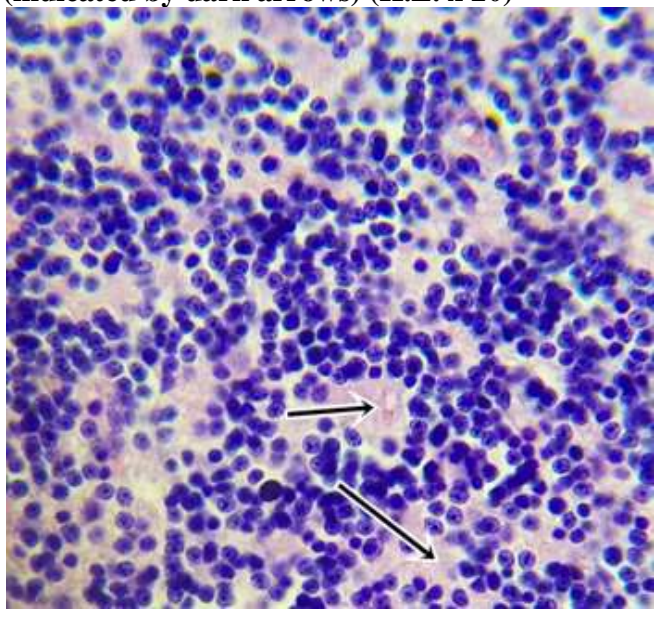

Figure no. 5. Several Homer-Wright rosettes - They are made up of tumor cells, placed evenly around a central area (indicated by dark arrows) (H.E x 40)

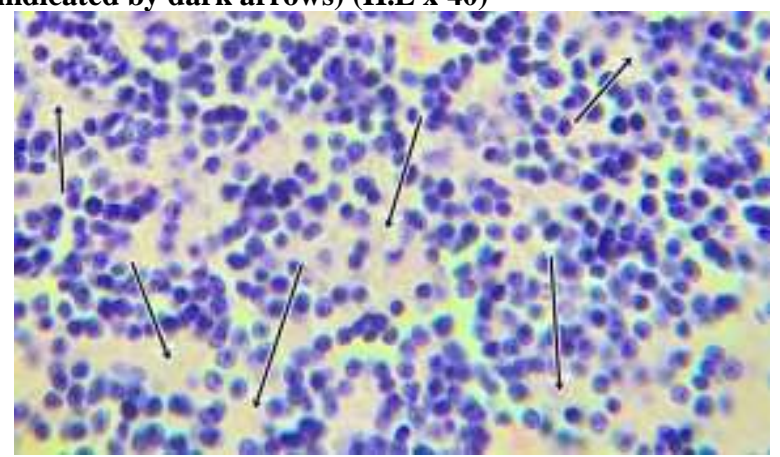

\section{CONCLUSIONS}

Neurological and imaging examination is essential and provides important details regarding the diagnosis and staging of the tumour, but histopathological examination remains the standard method that specifies the type (classical or undifferentiated, anaplastic, desmoplastic or nodular) of tumour formation. Histopathologically, the colour used for this type of preparation is hematoxylin - eosin.

Immunohistochemistry, the method that combines immunological tests with histological ones but also with biochemical tests, complements the histopathological examination using various antibodies. These extremely valuable markers are used to differentiate a medulloblastoma and other types of malignant tumours. In the present case, the following antibodies were analysed:

Ki67 - Is a nuclear protein associated with cell proliferation. It is an index of mitotic proliferation. In this case it is as a valuable prognostic biomarker for adult patients with medulloblastoma - $50 \%$,

Cytokeratin AE 1-3 - Is a mixture of antibodies, used to highlight the presence of metastases, being negative in this situation. It is used to exclude the presence of epithelial tumours.

Cytokeratin MNF116 - Is a monoclonal antibody. As with the previous marker, it is used to rule out the presence of epithelial tumours. It is negative in this case.

Vimentin - Confirms the mesenchymal origin in certain tumours. It is negative.

HMB45 or Human Melanoma Black - It is 


\section{CLINICAL ASPECTS}

monoclonal antibody, used as a marker for such tumours. Specifically, it is used to rule out the presence of melanocytic tumours. It is negative in this case.

S100 - Marker used in patients with distant metastases or in cases of nerve tissue tumours, but it is negative in this case.

Cytokeratin 20 - Protein commonly found in various types of carcinomas. Here we can mention colon or urothelium adenocarcinomas. In medulloblastoma this antibody is negative. It is negative.

Cytokeratin 5/6 - Markers for several types of cancer.

Cytokeratin 7 - It is a low molecular weight cytokeratin. It is used to exclude the presence of a lung adenocarcinoma. It is negative in this case.

Chromogranin - It is a secretory protein, negative in medulloblastoma. It is used to rule out the presence of neuroendocrine tumours.

TTF1 or Thyroid transcription factor-1 Multifunctional nucleolar protein. It is used to rule out the presence of a pulmonary adenocarcinoma. It is negative in medulloblastoma.

Histopathological aspect but also the immunohistochemical examinations performed show the presence of a cerebellar medulloblastoma, the classic type.

\section{REFERENCES}

1. Cooke RA, Stewart B. Colour Atlas of Anatomical Pathology. Third edition. Elsevier; 2004. p. 266.

2. Prayson RA, Cohen ML. Practical Differential Diagnosis in Surgical Neuropathology. Humana Press Inc.; 2000. p. 107112.

3. Haberland C. Clinical Neuropahology. Text and Color Atlas: Demos Medical Publishing; 2007.

4. Hart IJr. Tumors of the Fetus and Infant. Springer-Verlag New York, Inc.; 2002. p. 210-212.

5. Weidner N, Cote RJ, Suster S, Weiss LM. Modern Surgical Pathology: Saunders Elsevier; 2009.

6. Tonn J-C, Westphal M, Rutka JT, Grossman SA. NeuroOncology of CNS Tumors: Springer-Verlag Berlin Heidelberg; 2006.

7. Dabbs D. Diagnostic Immunohistochemistry. Third Edition: Saunders Elsevier; 2010.

8. Rose AG. Atlas of Gross Pathology with Histologic Correlation. Cambridge University Press; 2008. p. 648.

9. Kemp LK, Burns DK, Brown TG. Big Picture Pathology. The McGraw-Hill Companies, Inc.; 2008.

10. Riede UN, Werner M. Color Atlas of Pathology: Georg Thieme Verlag; 2004.

11. Cheng L, Bostwick DG. Essentials of Anatomic Pathology. Second Edition: Humana Press Inc.; 2006. p. 335.

12. Louis DN, Ohgaki H, Wiestler OD, Cavenee WK. WHO Classification of Tumours of the Central Nervous System: International Agency for Research on Cancer: Lyon; 2007.

13. Gatusso P, Reddy VB, David O, et all. Differential Diagnosis in Surgical Pathology. 2nd Edition: Saunders Elsevier; 2010.

14. Henson DE, Albores-Saavedra J. Pathology of Incipient Neoplasia. Third Edition: Oxford University Press; 2001.

15. Ali-Osman F. Brain Tumors: Humana Press Inc.; 2005.

16. Tadrous PJ. Diagnostic Criteria Handbook in Histopathology: A Surgical Pathology Vade Mecum: John Wiley \& Sons Ltd; 2007.

17. Damjanov I, Linder J. Pathology : A Color Atlas: Mosby; 1999.

18. Weedman Molavi D. The Practice of Surgical Pathology: A Beginner's Guide to the Diagnostic Process. Springer Science + Business Media; 2008.
19. Gray F, De Girolami U, Poirier J. Escourolle \& Poirier Manual of Basic Neuropathology. Fourth Edition: Elsevier Inc.; 2004.

20. Armstrong D, Halliday W, Hawkins C, Takashima S. Pediatric Neuropathology: A Text-Atlas. Springer; 2007. 